\title{
Influences on the quality of young children's diets: the importance of maternal food choices
}

\author{
Catherine M. Fisk ${ }^{1 *}$, Sarah R. Crozier ${ }^{1}$, Hazel M. Inskip ${ }^{1}$, Keith M. Godfrey ${ }^{1,2}$, Cyrus Cooper $^{1,2}$, \\ Siân M. Robinson ${ }^{1,2}$ and The Southampton Women's Survey Study Group \\ ${ }^{1}$ MRC Epidemiology Resource Centre, University of Southampton, Southampton General Hospital, Southampton \\ SO16 6YD, UK \\ ${ }^{2}$ NIHR Nutrition Biomedical Research Unit, Southampton University Hospitals NHS Trust, Southampton SO16 6YD, UK
}

(Received 26 January 2010 - Revised 23 July 2010 - Accepted 29 July 2010 - First published online 1 September 2010)

\section{Abstract}

It is recognised that eating habits established in early childhood may track into adult life. Developing effective interventions to promote healthier patterns of eating throughout the life course requires a greater understanding of the diets of young children and the factors that influence early dietary patterns. In a longitudinal UK cohort study, we assessed the diets of 1640 children at age 3 years using an interviewer-administered FFQ and examined the influence of maternal and family factors on the quality of the children's diets. To describe dietary quality, we used a principal components analysis-defined pattern of foods that is consistent with healthy eating recommendations. This was termed a 'prudent' diet pattern and was characterised by high intakes of fruit, vegetables and wholemeal bread, but by low intakes of white bread, confectionery, chips and roast potatoes. The key influence on the quality of the children's diets was the quality of their mother's diets; alone it accounted for almost a third of the variance in child's dietary quality. Mothers who had better-quality diets, which complied with dietary recommendations, were more likely to have children with comparable diets. This relationship remained strong even after adjustment for all other factors considered, including maternal educational attainment, BMI and smoking, and the child's birth order and the time spent watching television. Our data provide strong evidence of shared family patterns of diet and suggest that interventions to improve the quality of young women's diets could be effective in improving the quality of their children's diets.

\section{Key words: Diet quality: Dietary patterns: Pre-school children: Principal components analysis}

A growing body of evidence that links early experience with later health has focused attention on the importance of adequate nutrition in infancy and childhood $^{(1-3)}$. Health behaviours established in early life tend to 'track' from childhood to adult life ${ }^{(4,5)}$ and the stability of eating behaviours throughout childhood suggests that the ways in which children interact with their food environments may persist over time ${ }^{(6)}$. Interventions to promote healthy diets in early life may therefore have the potential to influence patterns of eating throughout the life course.

To develop effective interventions to improve children's diets, there is a need to understand more about the diets of young children and the factors that influence their eating patterns. Contemporary dietary data for young children in the UK are limited and relatively little is known about present variations in their dietary habits. More is known about variability in the quality of adult diets, which has been described in a number of studies. Poorer diets are more common in younger adults, those of lower educational attainment, smokers, those who watch more television (TV)/d, who have a lower income and who share their home with more children ${ }^{(7-9)}$. Analysis of British dietary data suggests that there are common influences on children's diets ${ }^{(10,11)}$. For example, children aged 1.5 to 4.5 years of age, who were studied between 1992 and 1993 as part of the National Diet and Nutrition Survey, were less likely to consume fruit and salad vegetables if they were from manual social classes and less advantaged households ${ }^{(11,12)}$. This is consistent with the findings from the Avon Longitudinal Study of Pregnancy and Childhood, which found poorer diets to be more common in 3-year-old children whose mothers reported a higher degree of financial difficulty and were of a lower educational attainment ${ }^{(10)}$.

We have previously described substantial variations in infant feeding practice among infants aged 6 and 12

Abbreviations: PCA, principal components analysis; SWS, the Southampton Women's Survey; TV, television.

*Corresponding author: C. M. Fisk, fax +44 23807040 21, email cmf@mrc.soton.ac.uk 
months in a large UK mother-offspring cohort, the Southampton Women's Survey (SWS) ${ }^{(13)}$. A key finding was that the most important predictor of infant's diets and their compliance with feeding guidance was the quality of their mother's diets. In the present study, we present the dietary data of the SWS children at the age of 3 years. We examine variations in dietary quality and we assess how this is influenced by a wide range of maternal and child characteristics, including the quality of the mother's diet.

\section{Methods}

\section{The Southampton Women's Survey}

The SWS is a large prospective cohort study that was started in $1998^{(14)}$. A total of 12583 non-pregnant women aged 20-34 years were recruited to the study. On enrolment, detailed information on diet and socio-demographic factors was collected. Children born to SWS women are followed up at home by trained research nurses at the ages of 6 and 12 months $^{(13)}$, and at 2 and 3 years and beyond. The SWS was approved by the Southampton and South West Hampshire Local Research Ethics Committee.

There were 1981 singleton live births to women in the SWS up to the end of 2003. After exclusion of infants with major congenital abnormalities ( $n$ 2) and neonatal deaths ( $n$ 6), there were 1973 SWS infants for postnatal follow-up. Data presented here are for 1640 (83\%) children, who were visited at 3 years of age by trained research nurses.

\section{Dietary assessment}

At the 3-year visit, the child's diet over the preceding 3 months, and information on eating behaviour, physical activity and illnesses was recorded at interview. For 1631 (99.5\%) children, the interview was conducted with the child's mother. Diet was assessed using an FFQ that was administered by trained research nurses. This FFQ was developed for use in the SWS using a variety of sources of dietary information. The list of foods and beverages to be included was compiled from a review of dietary intake data collected from a nationally representative sample of children aged 3 years between 1992 and $1993^{(12)}$, the SWS infants ${ }^{(13)}$ and adults ${ }^{(15)}$, and 3-yearolds in the Avon Longitudinal Study of Pregnancy and Childhood $^{(10)}$. Final amendments were made to the FFQ at the piloting stage of the present study. The FFQ asked about the average frequency of consumption of eighty food and beverage items in the preceding 3 months with eleven options that were (i) never, (ii) less than once/ month, (iii) 1-3 times/month, (iv-x) 1-7 times/week or (xi) more than once/d. If a food was consumed more than once a day, the number of times/d was recorded. At interview, prompt cards were used to show examples of the foods included in each food group and to help standardise the responses to the FFQ. Portion sizes were recorded; for bread, this was the number of slices, and for potatoes, the number of egg-sized potatoes consumed. At the end of the FFQ, additional information was collected relating to milk consumption and sugar added to food and/ or drinks each day. This included information on the type and quantity of milk consumed and the number of teaspoons of sugar added to the child's food and drinks. Frequencies of consumption and amounts of foods not listed in the FFQ were also recorded if they were consumed once a week or more.

\section{Dietary patterns of mothers and children}

Dietary patterns of the children at the age of 3 years were defined using principal components analysis (PCA) ${ }^{(16)}$, a multivariate statistical technique that objectively determines the axis along which diet varies the most. Before the PCA, the eighty foods listed on the 3-year FFQ were grouped into forty-four groups based on the similarity of food type and nutrient composition (for example, chocolate, sweets and ice lollies were included in the 'confectionery' group; carrots, parsnips, swede and turnips were included in the 'root vegetables' group). Milks (full fat and reduced fat) and sugar (added to food and drinks) were put into three extra groups to give a total of fortyseven groups. Most additional foods recorded at the end of the FFQ were assigned to these forty-seven groups. The remaining foods did not fit into these groups and four further groups (baby foods, fruit purées, cream and Yorkshire pudding and savoury pancakes) were created for these. This gave a total of fifty-one groups to be entered into the 3-year PCA.

PCA was performed on the reported weekly frequencies of consumption of forty-four of the fifty-one food groups, and the reported weekly amounts consumed per serving of the remaining seven food groups (boiled potatoes, chips and roast potatoes, white bread, brown bread, sugar, reduced-fat milk and full-fat milk). It was based on the correlation matrix in order to adjust for unequal variances of the original variables. The first component from the PCA explained $7 \cdot 5 \%$ of the variance in the fifty-one food groups and yielded a dietary pattern that was characterised by high intakes of fruit and vegetables, but by low intakes of white bread and crisps. This pattern was termed a 'prudent' diet pattern, as it represented a diet consistent with healthy eating recommendations and was similar to the 'prudent' pattern that we have previously described in the SWS women ${ }^{(9)}$. The second and third components identified from the PCA were more difficult to interpret and explained less of the variance in the fifty-one food groups ( 4.6 and $3.7 \%$, respectively) ${ }^{(17)}$. The second component was characterised by a high frequency of consumption of foods that resemble a more 'traditional' pattern of eating, including red meat, puddings and green vegetables. 
The third component was characterised by a high frequency of consumption of vegetable dishes and vegetarian foods, quiche and pizza and beans and pulses ${ }^{(17)}$. In the present paper, we considered only the prudent diet pattern, as it was the most meaningful and interpretable pattern of foods, and it provided an index of the quality of the child's diet.

For each child, a prudent diet score was calculated based on the reported frequency of consumption of the fifty-one food groups and the fifty-one coefficients produced by PCA for the first component. The scores were then standardised to have zero mean and unit variance using FisherYates normal scores ${ }^{(18)}$. High prudent diet scores represent frequent consumption of foods with positive coefficients and less frequent consumption of foods with negative coefficients, and indicate diets that more closely conform to healthy eating recommendations. The prudent diet score was used as a continuous variable to indicate compliance with the prudent diet pattern.

We have previously described the dietary patterns of the women in the SWS ${ }^{(19)}$. Before pregnancy, on enrolment to the study, diet was assessed using an $\mathrm{FFQ}^{(15)}$ (median interval between dietary assessment and the birth of the child was $20 \cdot 8$ (interquartile range $13 \cdot 9-30 \cdot 2$ ) months). Using PCA, we identified the key dietary pattern as a 'prudent' pattern that was characterised by high intakes of fruit, vegetables, wholemeal bread, rice and pasta, but by low intakes of chips and roast potatoes, sugar and red and processed meat. A prudent diet score, indicating compliance with the prudent pattern, was available for each mother.

\section{Maternal and 3-year-old offspring data}

On enrolment to the study, we recorded the mother's age, educational attainment, number of children, ethnicity and whether she lived with a partner or not. Educational attainment was defined in six groups according to the women's highest academic qualification: none, General Certificate of Secondary Education grade D or below, General Certificate of Secondary Education grade C or above, Advanced Level or equivalent, Higher National Diploma or equivalent and degree. Examinations for General Certificate of Secondary Education are usually taken at the age of 16 years, Advanced Levels at 18 years, and Higher National Diploma and degrees thereafter. Birth order was defined in four groups using the number of children: 0 (first born), 1 (second born), 2 (third born) and 3 or more (fourth or later born). A deprivation score was also calculated for the address at which each woman lived (Index of Multiple Deprivation 2004) ${ }^{(20)}$. When the child was 6 months of age, data were collected on maternal smoking status and maternal BMI was measured. Social class of the mother was defined in six groups according to the woman's employment when the child was 2 years of age ${ }^{(21)}$. At 3 years of age, the child's BMI was measured and the time spent watching $\mathrm{TV} / \mathrm{d}$ was recorded. The parents were asked to indicate the number of times in a day the child ate (including meals, snacks, biscuits with drinks, etc) and to assess their nibbling behaviour. Nibbling behaviour was categorised into four groups: nibbles during the day, rarely eats meals; nibbles during the day but also has meals; nibbles on some days but also has meals; doesn't nibble much, just has meals. The short form of the Household Food Security Scale was included in the questionnaire and consisted of six questions that were used to determine a score for classifying the food security of households ${ }^{(22)}$. These six questions asked about the perceived affordability of food in the household; for example, whether the respondents ever ate less than they wished because there was not enough money to buy food.

\section{Statistical analysis}

Data were analysed using Stata version 10.0 (StataCorp LP, College Station, TX, USA). We investigated the associations between the 3-year prudent diet score and the following sixteen maternal and family factors: maternal prudent diet score, maternal age, maternal educational attainment, child's birth order, ethnicity of the child, sex of the child, whether the mother lived with a partner or not, household deprivation score, maternal smoking status, maternal BMI, maternal social class, child's BMI, time spent watching TV at 3 years, how many times a day the child ate (including meals, snacks, biscuits with drinks, etc), the child's nibbling behaviour and household food security. Multiple regression analysis, using the backwards elimination method, was used to identify the most important influences on the prudent diet score at 3 years. Resultant regression $(\beta)$ coefficients, associated $95 \% \mathrm{CI}$ and $t$ statistics were used to assess the strength of association between these factors and the prudent diet score.

\section{Results}

The characteristics of both the women and children studied are shown in Table 1 . The child's average age at interview was 3 years and 3 weeks (interquartile range 3 years and 1 week to 3 years and 6 weeks). A total of $192(12 \%)$ and 41 (3\%) 3-year-olds were overweight and obese, respectively, according to International Obesity Task Force cut-offs at 3 years of age ${ }^{(23)}$.

Table 2 shows the coefficients ranked in order for the fifty-one food groups for the prudent diet pattern. The pattern was characterised by high intakes of fruit, vegetables, water, wholemeal bread, fish, and fruit juices, but by low intakes of white bread, crisps, chips and roast potatoes, processed meat, confectionery and cakes and biscuits.

\section{Prudent diet score and food intake}

Table 3 shows the 3-year-olds grouped into quarters according to their prudent diet score. The median weekly 
Table 1. Characteristics of the women and 3-year-olds

(Number of subjects and percentage or median values and interquartile ranges (IQR))

\begin{tabular}{|c|c|c|c|c|}
\hline Characteristics & $n$ & $\%$ & Median & IQR \\
\hline \multicolumn{5}{|l|}{ Women ( $n$ 1640) } \\
\hline Age (years) when child was 3 years old & & & 33.5 & $30 \cdot 5-36 \cdot 2$ \\
\hline BMI $\left(\mathrm{kg} / \mathrm{m}^{2}\right) \dagger$ & & & $25 \cdot 6$ & $23 \cdot 0-29 \cdot 2$ \\
\hline \multicolumn{5}{|l|}{ Educational attainment ${ }^{*}$} \\
\hline None & 44 & $2 \cdot 7$ & & \\
\hline GCSE grade $\mathrm{D}$ or below & 163 & $10 \cdot 0$ & & \\
\hline GCSE grade $\mathrm{C}$ or above & 476 & $29 \cdot 1$ & & \\
\hline A-level or equivalent & 484 & $29 \cdot 6$ & & \\
\hline HND or equivalent & 117 & $7 \cdot 2$ & & \\
\hline Degree & 352 & 21.5 & & \\
\hline Smokers $\dagger$ & 300 & 18.5 & & \\
\hline \multicolumn{5}{|l|}{ Ethnicity* } \\
\hline White & 1567 & $95 \cdot 6$ & & \\
\hline Other & 73 & 4.5 & & \\
\hline Living with a partner - yes* & 1346 & $82 \cdot 1$ & & \\
\hline \multicolumn{5}{|l|}{ Social class $\ddagger$} \\
\hline Manual & 324 & $20 \cdot 2$ & & \\
\hline Non-manual & 1277 & $79 \cdot 8$ & & \\
\hline \multicolumn{5}{|l|}{ 3-Year olds $(n 1640)$} \\
\hline BMI $\left(\mathrm{kg} / \mathrm{m}^{2}\right)$ & & & $16 \cdot 3$ & $15 \cdot 4-17 \cdot 1$ \\
\hline Sex - boys & 870 & $53 \cdot 1$ & & \\
\hline \multicolumn{5}{|l|}{ Birth order } \\
\hline First & 759 & $46 \cdot 3$ & & \\
\hline Second & 632 & 38.6 & & \\
\hline Third & 178 & $10 \cdot 9$ & & \\
\hline Fourth or later & 70 & 4.3 & & \\
\hline \multicolumn{5}{|l|}{ Time spent watching TV/d (h) } \\
\hline 0 & 10 & 0.6 & & \\
\hline$<1$ & 289 & 18.5 & & \\
\hline $1-2$ & 666 & $42 \cdot 7$ & & \\
\hline $2-5$ & 590 & $37 \cdot 8$ & & \\
\hline $5+$ & 5 & 0.3 & & \\
\hline \multicolumn{5}{|l|}{ Food security } \\
\hline Food secure & 1543 & 95.4 & & \\
\hline Food insecure without hunger & 52 & $3 \cdot 2$ & & \\
\hline Food insecure with hunger & 23 & 1.4 & & \\
\hline \multicolumn{5}{|l|}{ Eating behaviour } \\
\hline Nibbles during the day, rarely eats meals & 68 & 4.2 & & \\
\hline Nibbles during the day, but also has meals & 759 & $46 \cdot 4$ & & \\
\hline Nibbles on some days, but also has meals & 564 & 34.5 & & \\
\hline Does not nibble much, just has meals & 244 & 14.9 & & \\
\hline \multicolumn{5}{|c|}{ How many times a day the child eats, including meals, snacks, biscuits with drinks, etc } \\
\hline $1-3$ & 86 & $5 \cdot 3$ & & \\
\hline 4 & 371 & $22 \cdot 7$ & & \\
\hline 5 & 718 & $43 \cdot 8$ & & \\
\hline 6 & 334 & $20 \cdot 4$ & & \\
\hline $7+$ & 129 & 7.9 & & \\
\hline
\end{tabular}

GCSE, General Certificate of Secondary Education; A-level, Advanced Level; HND, Higher National Diploma, TV, television.

* Data collected on the woman's enrolment to the study.

† Data collected when the child was 6 months of age.

$\ddagger$ Data collected when the child was 2 years of age.

consumption of some of the most important food groups that characterised the prudent diet pattern is shown for the 3-year-olds according to their prudent diet score. Increasing scores were associated with graded increases in the consumption of vegetables, non-citrus fruits, water, wholemeal bread and fish and shellfish, but with graded decreases in the consumption of cakes and biscuits, confectionery, processed meat, chips and roast potatoes, crisps and white bread. The differences in consumption of particular foods were large. For example, when fruit and vegetables were combined, $0.03 \%$ of 3 -year-olds with prudent diet scores in the bottom quarter of the distribution consumed fruit and vegetables five or more times a day, compared with $58 \%$ of 3 -year-olds who had scores in the top quarter (data not shown).

\section{Associations between diet quality and maternal and family characteristics}

The associations between 3-year prudent diet score and sixteen maternal and child factors were investigated. With the exception of the child's sex, significant associations were found with fifteen of these factors. Children with higher prudent diet scores were more likely to have 
Table 2. Principal components analysis coefficients for the prudent diet pattern

\begin{tabular}{lc}
\hline & \\
Food or food group & Component 1 \\
coefficients \\
\hline Green vegetables & 0.26 \\
Root vegetables & 0.25 \\
Water & 0.23 \\
Non-citrus fruit & 0.23 \\
Salad vegetables & 0.23 \\
Wholemeal bread (slices) & 0.22 \\
Dried fruit & 0.19 \\
Fish and shellfish & 0.19 \\
Fruit juices & 0.17 \\
Vegetable dishes and vegetarian foods & 0.16 \\
Rice and pasta & 0.16 \\
Crackers & 0.14 \\
Other vegetables & 0.14 \\
Cooked and tinned fruit & 0.12 \\
Chicken and turkey & 0.12 \\
Nuts and seeds & 0.11 \\
Cheese and cottage cheese & 0.11 \\
Baby foods & 0.10 \\
Citrus fruit & 0.10 \\
Eggs and egg dishes & 0.08 \\
Beans and pulses & 0.07 \\
Red meat & 0.07 \\
Breakfast cereals & 0.07 \\
Marmite and Bovril & 0.06 \\
Sweet spreads & 0.06 \\
Fruit purées & 0.05 \\
Boiled potatoes (egg sized) & 0.05 \\
Soup & 0.04 \\
Full-fat milk (pints) & 0.04 \\
Cream & 0.04 \\
Reduced-fat spread (teaspoons) & 0.03 \\
Sauces and salad dressings & 0.01 \\
Offal & 0.01 \\
Reduced-fat milk (pints) & 0.00 \\
Yorkshire pudding and savoury pancakes & -0.02 \\
Quiche and pizza & -0.02 \\
Puddings & -0.03 \\
Yoghurt & -0.04 \\
Full-fat spread (teaspoons) & -0.05 \\
Milky drinks & -0.08 \\
High-energy soft drinks & -0.08 \\
Tea and coffee & -0.11 \\
Added sugar (teaspoons) & -0.12 \\
Tinned vegetables & -0.12 \\
Cakes and biscuits & -0.13 \\
Confectionery & -0.18 \\
Low-energy soft drinks & -0.19 \\
Processed meat & -0.19 \\
Chips and roast potatoes (egg sized) & -0.23 \\
White bread (slices) & -0.25 \\
\hline & -0.26 \\
Variance explained (\%) & \\
\hline
\end{tabular}

mothers with a higher prudent diet score, who were older, had a higher level of educational attainment, were of a lower BMI, were non-smokers, living with a partner, who were white Caucasian and who were of a higher social class (all $P<0 \cdot 001$ ). Higher prudent diet scores in the 3-year-olds were also associated with a lower birth order $(P<0.001)$, watching less TV/d $(P<0.001)$, living in a food-secure household ( $P=0.007)$, eating more meals compared with nibbling $(P<0 \cdot 001)$, eating fewer times a day (including meals, snacks, biscuits with drinks, etc)
$(P=0 \cdot 003)$, living in a less-deprived household $(P<0 \cdot 001)$ and having a lower BMI $(P=0.035)$.

Since many of these factors were related, we then explored the factors which were independently associated with the 3-year prudent diet score by entering them into a multiple regression model. Table 4 shows that eight factors remained independently associated with the score, and they collectively explained $40.9 \%$ of the variation. However, on its own, the mother's prudent diet score explained $30.5 \%$ of the variance in the 3 -year prudent diet score. This effect was strong and graded; for example, of mothers with the lowest prudent diet scores (in the lowest sixth of the distribution), $62 \%$ had 3-year olds with low prudent diet scores (in the lowest quarter of the distribution), but only $0.05 \%$ had 3-year-olds with high prudent diet scores (in the highest quarter of the distribution) (data not shown). The correlation between maternal and child prudent diet scores was high $(r 0.55, P<0.001)$ such that $74 \%$ of both the prudent diet pattern scores were within $1 \mathrm{SD}$ of each other. In the multivariate analysis food security, social class, child's BMI, number of meals consumed/d (including meals, snacks, biscuits with drinks, etc), whether the mother lived with a partner or not, ethnicity and maternal age no longer had independent influences on the 3-year prudent diet score.

Fig. 1 illustrates the key influences on the 3-year prudent diet score. It shows the proportion of 3-year-olds with the poorest-quality diets (with prudent diet scores in the lowest quarter of the distribution) according to maternal prudent diet score. The four graphs show the strong and graded influence of the quality of the mother's diet on the quality of the child's diet. In addition to the effects seen with maternal prudent diet score, the smaller effects of maternal educational attainment, birth order, TV watching in the child and nibbling behaviour are also shown.

\section{Discussion}

We have shown wide variation in the diets of a general population sample of 3-year-olds living in Southampton, UK. The quality of the children's diets was strongly related to a number of maternal and family characteristics, but the most important was the quality of the mother's diet. On its own, this accounted for almost a third of the variance in the quality of diet at 3 years.

\section{Dietary patterns in the 3-year-olds}

We used PCA to identify the key dietary pattern that we termed 'prudent' as it was consistent with 'prudent' and 'healthy' dietary patterns derived using factor analysis in previous studies ${ }^{(10,13,19,24,25)}$. Three-year-olds with a high score for this pattern had diets that were characterised by high intakes of fruits and vegetables, wholemeal bread, rice and pasta and fish and shellfish. Children with a low diet score had diets characterised by lower intakes of the 


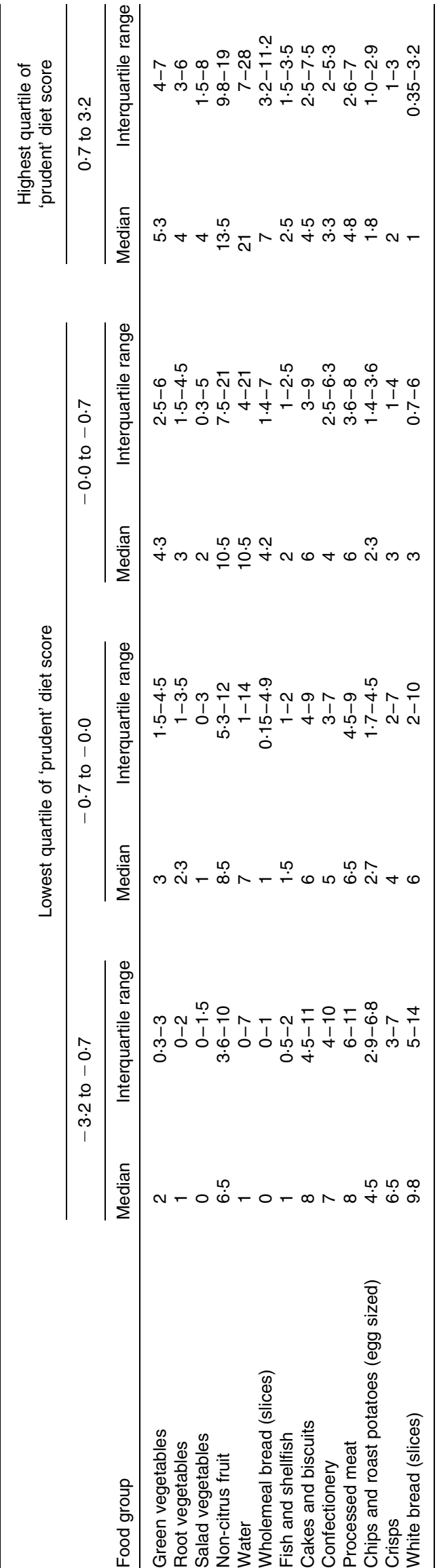

above foods and higher intakes of white bread, chips and roast potatoes, processed meat and crisps. The child's pattern score indicated their compliance with the pattern and we used it as an index of diet quality. In the SWS, we have previously used PCA and derived comparable dietary patterns in both the infants and women that were consistent with healthy eating recommendations ${ }^{(9,13)}$. At 6 and 12 months, the first components derived using PCA were similarly characterised by a high frequency of consumption of vegetables and fruit ${ }^{(13)}$. Our findings are also consistent with other studies of young children outside the UK. For example, similar eating patterns which represented a measure of how healthy the diet was have been identified in Korean ${ }^{(26)}$ and Spanish ${ }^{(24)}$ children, using factor analysis. Using cluster analysis, Ovaskainen et $a l .{ }^{(27)}$ defined a 'healthy' cluster in 3-year-old Finnish children that was characterised by a high consumption of whole-grain bread and vegetables. These a posteriori methods have therefore defined 'healthy' dietary patterns that are comparable to the Revised Children's Diet Quality Index, an a priori-defined pattern based on eating recommendations, and used to assess the diets of American preschoolers. High scores for this index represented diets with higher intakes of fibre, essential fatty acids, fruits and vegetables $^{(28)}$.

\section{Family influences on 3-year-olds' diets}

Better-quality diets were more common in children who had mothers with a higher prudent diet score, who had reached a higher level of educational attainment, who lived in a less-deprived area and who were of a lower BMI and non-smokers. Children who were of a lower birth order, who spent less time watching TV/d and ate more meals in comparison with nibbling throughout the day were also more likely to be consuming a more prudent diet. By far, the most important determinant of the child's diet was the quality of the mother's diet ${ }^{(19)}$. Children of mothers who had a prudent diet with high intakes of fruit, vegetables and wholemeal bread were likely to have a comparable diet at 3 years of age. Conversely, children of mothers who had diets characterised by high intakes of foods such as white bread, crisps and confectionery were more likely to have poorer-quality diets at 3 years of age. This is consistent with earlier findings in this cohort of strong associations between the maternal prudent diet pattern and a pattern showing compliance with infant feeding guidelines at 6 and 12 months of age $^{(13)}$. However, the percentage variance in diet score explained by the mother's diet quality was stronger at 3 years compared with that in infancy ( $31 \%$ at 3 years, compared with $24 \%$ at 12 months) (CM Fisk, unpublished results). This might be expected as beyond infancy, as the young child grows older, its diet becomes more based on foods that are consumed by the family. Other studies have found similar associations between parents' 
Table 4. Multiple regression analysis of the factors independently associated with 'prudent diet' score at 3 years" (Regression coefficients, $95 \%$ confidence intervals and $t$ statistics $(\beta)$ )

\begin{tabular}{lccrr}
\hline Characteristics $\dagger$ & $\beta$ & $95 \% \mathrm{Cl}$ for $\beta$ & $t$ Statistic & $P$ \\
\hline Mother & & & & \\
$\quad$ Prudent diet score & 0.39 & $0.34,0.43$ & 16.51 & $<0.001$ \\
Educational attainment & 0.11 & $0.08,0.15$ & 6.61 & $<0.001$ \\
Maternal BMI & -0.06 & $-0.09,-0.02$ & -2.75 & 0.006 \\
Smoking status & -0.11 & $-0.22,-0.00$ & -2.04 & 0.041 \\
$\quad$ Index of multiple deprivation & -0.008 & $-0.01,-0.004$ & -4.27 & $<0.001$ \\
3-Year old & & & -6.43 & $<0.001$ \\
Birth order & -0.17 & $-0.22,-0.12$ & -5.59 & $<0.001$ \\
Time spent watching TV/d (h) & -0.11 & $-0.15,-0.07$ & 3.80 & $<0.001$ \\
Nibbling behaviour & 0.10 & $0.05,0.15$ & & \\
\hline
\end{tabular}

TV, television.

*Variance explained by these factors $=40.9 \%$.

†All of the characteristics have been adjusted for each other in the multivariate analysis.

and young children's diets ${ }^{(29-35)}$. For example, Cooke et al. ${ }^{(30)}$ found that the amount of vegetables that parents themselves reported eating was a strong predictor of their 2-6-year-old child's intake of vegetables $(r$ 0.49, $P<0 \cdot 001)$. A study by Vereecken $e t$ al. ${ }^{(34)}$ also found that maternal consumption was a strong predictor of the intake of fruit, vegetables, soft drinks and sweets in children aged $2 \cdot 5$ to 7 years. These similarities in food consumption translate into correspondence in nutrient intake in parents and children. For example, Oliveria et al. ${ }^{(33)}$ found a significant correlation between parents' and children's intakes for most nutrients using four sets of $3 \mathrm{~d}$

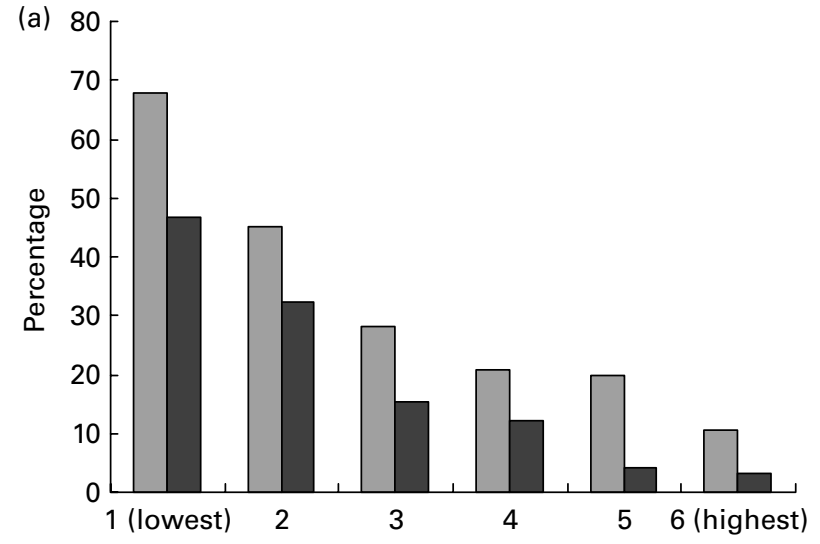

Sixths of maternal prudent diet score

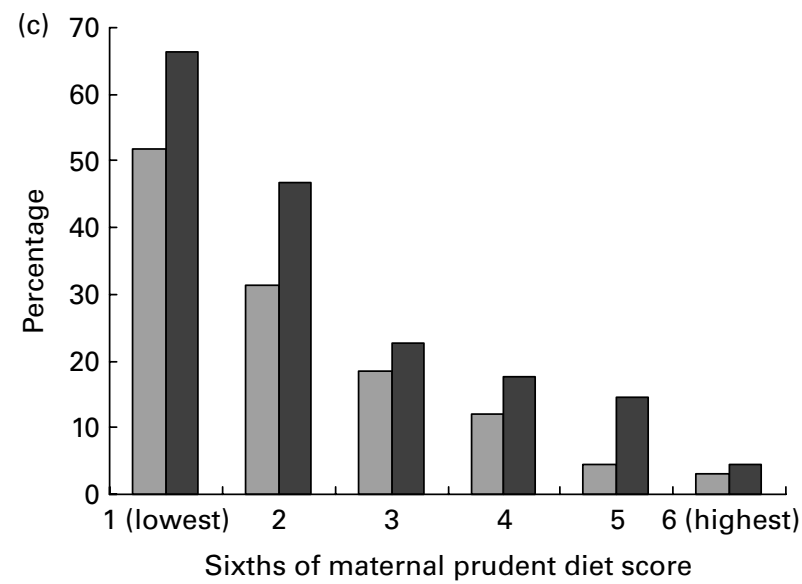

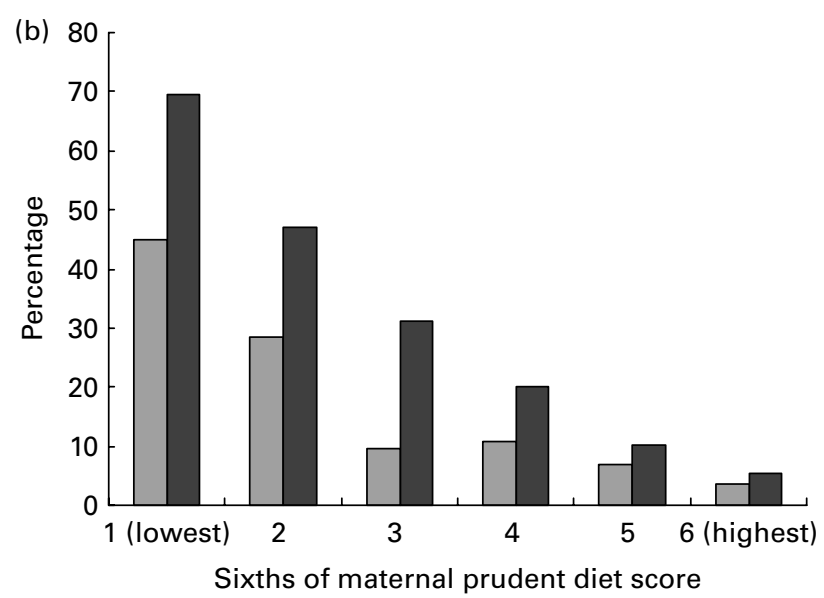

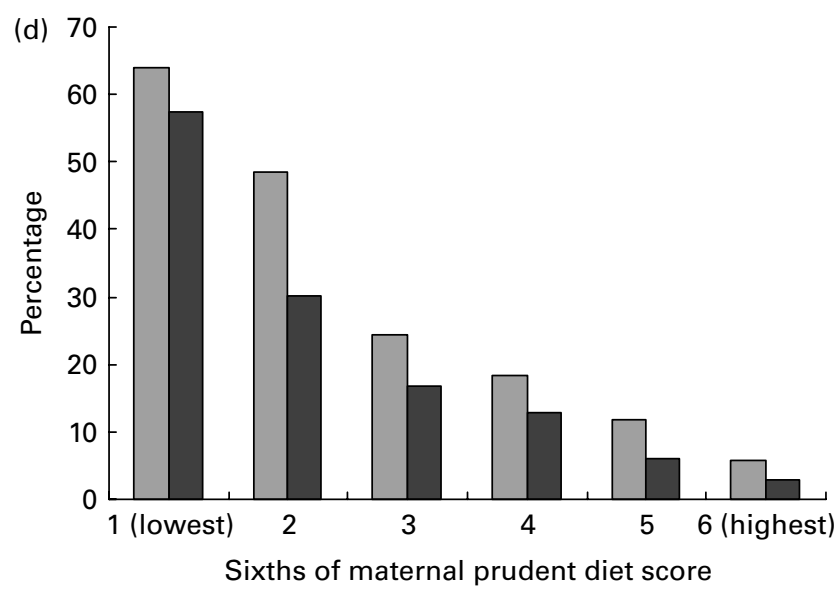

Fig. 1. Percentage of the 1640 3-year-olds, who are in the lowest quarter of the 3-year prudent diet score according to differences in maternal educational attainment (a) ( $\square$, percentage of mothers educated up to General Certificate of Secondary Education; $\square$, percentage of mothers educated to Advanced Level or above), birth order (b) ( $\square$, first born; $\square$, second or later born), time (h) spent watching television (TV)/d (c) ( $\square$, children watching $0-2 h$ of TV/d; $\square$, children watching more than $2 \mathrm{~h}$ of TV/d) and nibbling behaviour (d) ( $\square$, nibbles during the day; $\square$, nibbles infrequently, mainly eats meals), analysed within sixth of the distribution of maternal prudent diet score. 
diet records collected over a 1 -year period for mothers, fathers and their children aged 3-5 years.

Although maternal prudent diet was the strongest influence, a number of other maternal and family characteristics were found to influence the 3-year-olds' diet quality. Some of these characteristics, including educational attainment, have also been shown to influence adult diets ${ }^{(7-9)}$. The present findings are consistent with those from the Avon Longitudinal Study of Pregnancy and Childhood, in which poorer diets in 3-year-olds, characterised by a high consumption of foods such as chocolate, sweets, chips and fried food, were more common in children who had younger mothers, who were of a lower educational attainment and who reported a greater degree of financial difficulty ${ }^{(10)}$. In the SWS, the mother's educational attainment was the most influential factor in relation to the quality of her own diet and also a strong influence on her infant's diet at 6 and 12 months ${ }^{(9,13)}$. Although the mother's own food choices are the primary influence at 3 years of age, we have shown that educational attainment also remains an important determinant of the diet quality of her child.

We found a graded association between birth order and 3-year prudent diet score; 3-year-olds with fewer older siblings were more likely to have a diet conforming to present healthy eating recommendations. This is consistent with findings from the Avon Longitudinal Study of Pregnancy and Childhood study, which showed that children who had one or more older siblings were more likely to have a 'junk' dietary pattern compared with children who had no older siblings ${ }^{(10)}$. Having older siblings may result in the introduction of unhealthy foods, such as sugary snacks, into the home that would not have been present otherwise. SWS mothers have previously been shown to have a poorer-quality diet if they have more children in their home, and it is of concern that being part of a larger family may lead to the consumption of poorer diets in the children as well as the mother ${ }^{(9)}$. Watching less TV was also associated with better-quality diets in the 3-year-olds. Consistent with this finding, TV watching has been shown to be associated with a lower consumption of fruits and vegetables in a study by Coon et al. ${ }^{(36)}$. Strong associations have previously been found between TV watching and risk of obesity in children ${ }^{(37)}$. However, in our cross-sectional analyses, we did not find an association between the quality of the 3-year-olds' diets and child BMI.

Other factors that were related to better-quality diets in the children included living in a less-deprived area and having mothers who were of a lower BMI and nonsmokers. In line with these findings, analyses of the diets of children in the National Diet and Nutrition Survey show that children aged $1.5-4.5$ years from less-advantaged households were less likely to consume healthy foods, including fruit and salad vegetables ${ }^{(11)}$. We also found that dietary quality was related to the pattern of meal consumption throughout the day; children who consumed more meals in comparison with nibbling were more likely to have better-quality diets. A recent UK study showed an overall preference in children aged 7-8 years of age for unhealthy snacks such as sweets and crisps compared with more healthy snacks such as those containing fruit and cereal ${ }^{(38)}$. Children who nibble more may therefore be consuming a higher proportion of unhealthy foods as snacks. Since they do not eat meals as frequently, they are unlikely to be consuming foods such as vegetables that are predominantly prepared for consumption at mealtimes.

\section{Strengths and weaknesses}

We assessed diet in the 3-year-olds using an FFQ that was administered by trained research nurses. Although FFQ may be prone to bias ${ }^{(39)}$, they have been shown to describe similar dietary patterns to 1 -week diet records and $24 \mathrm{~h}$ recalls ${ }^{(40)}$, and in adults, the prudent pattern assessed by FFQ is predictive of a number of biomarkers, including erythrocyte folate and $\beta$-carotene concentrations ${ }^{(19,40)}$. The 1640 three-year-olds studied represented $83 \%$ of the SWS cohort; they are from a wide range of backgrounds and had many characteristics that were comparable with the wider UK population ${ }^{(14,41)}$. Our findings should therefore have relevance beyond Southampton. The mothers' dietary data were collected before pregnancy and maternal diet may have changed since then. However, we have previously shown the dietary patterns in SWS women to be reasonably stable over a 2 -year period and we would therefore not expect their diets to have changed considerably since assessment ${ }^{(42)}$. The strength of association between mothers' and children's dietary patterns and consistency with our earlier observations in infancy suggest that dietary change would not explain the associations we observed. The method of PCA is itself subject to a number of strengths and limitations. One possible limitation is that the researcher is required to make several subjective decisions on how to group the foods and how many components to retain, although we have previously shown that prudent pattern scores based on individual foods or food groups are highly correlated ${ }^{(19)}$. An advantage of this method is that it takes into account the co-linearity of foods and yields a pattern representing combinations of foods eaten together, providing information about the whole diet.

\section{Conclusion}

We have identified a number of maternal and family characteristics that are strongly associated with diet quality in pre-school children. These may provide insights into the key influences on children's diets and may enable identification and targeting of those children who are most in need of interventions to improve their diets. The strong association found between 3-year-olds and maternal diet 
quality suggests that interventions that focus on improving the mother's diet may also have an effect on the child's diet quality. As eating behaviours have shown stability throughout childhood $^{(6)}$ and dietary patterns may track from childhood to adulthood ${ }^{(4,5)}$, interventions to promote healthy diets and improve nutrient intake in early life may have the potential to influence the patterns of eating throughout the life course.

\section{Acknowledgements}

We thank all the families who participated in the SWS, and the SWS research staff and computing staff. H. M. I., K. M. G., S. M. R. and C. C. designed and ran the Southampton Women's Survey. The data were analysed by C. M. F. and S. R. C. All the authors contributed to the interpretation of the data, and C. M. F. and S. M. R. wrote the first draft of the manuscript. None of the authors had a conflict of interest. The present study was supported by the Medical Research Council, University of Southampton, British Heart Foundation, Food Standards Agency (contract N05071) and University of Southampton Research Policy Committee.

\section{References}

1. Magarey AM, Daniels LA, Boulton TJ, et al. (2003) Predicting obesity in early adulthood from childhood and parental obesity. Int J Obes Relat Metab Disord 27, 505-513.

2. Singhal A, Cole TJ, Fewtrell M, et al. (2004) Breastmilk feeding and lipoprotein profile in adolescents born preterm: follow-up of a prospective randomised study. Lancet 363 , 1571-1578.

3. Singhal A, Cole TJ \& Lucas A (2001) Early nutrition in preterm infants and later blood pressure: two cohorts after randomised trials. Lancet 357, 413-419.

4. Mikkila V, Rasanen L, Raitakari OT, et al. (2005) Consistent dietary patterns identified from childhood to adulthood: the cardiovascular risk in Young Finns Study. Br J Nutr 93 , 923-931.

5. Northstone K \& Emmett PM (2008) Are dietary patterns stable throughout early and mid-childhood? A birth cohort study. Br J Nutr 100, 1069-1076.

6. Ashcroft J, Semmler C, Carnell S, et al. (2008) Continuity and stability of eating behaviour traits in children. Eur J Clin Nutr 62, 985-990.

7. Dynesen AW, Haraldsdottir J, Holm L, et al. (2003) Sociodemographic differences in dietary habits described by food frequency questions - results from Denmark. Eur J Clin Nutr 57, 1586-1597.

8. Groth MV, Fagt S \& Brondsted L (2001) Social determinants of dietary habits in Denmark. Eur J Clin Nutr 55, 959-966.

9. Robinson SM, Crozier SR, Borland SE, et al. (2004) Impact of educational attainment on the quality of young women's diets. Eur J Clin Nutr 58, 1174-1180.

10. North K \& Emmett P (2000) Multivariate analysis of diet among three-year-old children and associations with sociodemographic characteristics. The Avon Longitudinal Study of Pregnancy and Childhood (ALSPAC) Study Team. Eur J Clin Nutr 54, 73-80.
11. Scientific Advisory Committee on Nutrition (2008) TheNutritional Wellbeing of the British Population. http:// www.sacn.gov.uk/pdfs/nutritional_health_of_the_population_ final_oct_08.pdf

12. Gregory J, Collins D, Davies PSW, et al. (2009) National Diet and Nutrition Survey children aged 1 1/2 to 4 1/2 years. Vol. 1: Report of the Diet and Nutrition Survey. London: HMSO.

13. Robinson S, Marriott L, Poole J, et al. (2007) Dietary patterns in infancy: the importance of maternal and family influences on feeding practice. Br J Nutr 98, 1029-1037.

14. Inskip HM, Godfrey KM, Robinson SM, et al. (2006) Cohort profile: the Southampton Women's Survey. Int J Epidemiol 35, 42-48.

15. Robinson S, Godfrey K, Osmond C, et al. (1996) Evaluation of a food frequency questionnaire used to assess nutrient intakes in pregnant women. Eur J Clin Nutr $\mathbf{5 0}$, 302-308.

16. Joliffe IT \& Morgan BJ (1992) Principal component analysis and exploratory factor analysis. Stat Methods Med Res 1, 69-95.

17. Fisk CM, Crozier SR, Inskip HM, et al. (2009) Dietary patterns in children: results from the Southampton Women's Survey. Proc Nutr Soc 69, E54.

18. Armitage P \& Berry G (2002) Statistical Methods in Medical Research. Oxford: Blackwell Science Ltd.

19. Crozier SR, Robinson SM, Borland SE, et al. (2006) Dietary patterns in the Southampton Women's Survey. Eur J Clin Nutr 60, 1391-1399.

20. Office of the Deputy Prime Minister (2004) The English Indices of Deprivation 2004 (revised). http://www.communities. gov.uk/documents/communities/pdf/131209.pdf

21. Office of Population Censuses Survey (1990) Standard Occupational Classification. London: HMSO.

22. Blumberg SJ, Bialostosky K, Hamilton WL, et al. (1999) The effectiveness of a short form of the Household Food Security Scale. Am J Public Health 89, 1231-1234.

23. Cole TJ, Bellizzi MC, Flegal KM, et al. (2000) Establishing a standard definition for child overweight and obesity worldwide: international survey. BMJ 320, 1240-1243.

24. Aranceta J, Perez-Rodrigo C, Ribas L, et al. (2003) Sociodemographic and lifestyle determinants of food patterns in Spanish children and adolescents: the enKid study. Eur J Clin Nutr 57, Suppl. 1, S40-S44.

25. Hu FB, Rimm EB, Stampfer MJ, et al. (2000) Prospective study of major dietary patterns and risk of coronary heart disease in men. Am J Clin Nutr 72, 912-921.

26. Shin KO, Oh SY \& Park HS (2007) Empirically derived major dietary patterns and their associations with overweight in Korean preschool children. Br J Nutr 98, 416-421.

27. Ovaskainen ML, Nevalainen J, Uusitalo L, et al. (2009) Some similarities in dietary clusters of pre-school children and their mothers. Br J Nutr 102, 443-452.

28. Kranz S, Hartman T, Siega-Riz AM, et al. (2006) A diet quality index for American preschoolers based on current dietary intake recommendations and an indicator of energy balance. J Am Diet Assoc 106, 1594-1604.

29. Brown R \& Ogden J (2004) Children's eating attitudes and behaviour: a study of the modelling and control theories of parental influence. Health Educ Res 19, 261-271.

30. Cooke LJ, Wardle J, Gibson EL, et al. (2004) Demographic, familial and trait predictors of fruit and vegetable consumption by pre-school children. Public Health Nutr 7, 295-302.

31. Fisher JO, Mitchell DC, Smiciklas-Wright H, et al. (2002) Parental influences on young girls' fruit and vegetable, micronutrient, and fat intakes. J Am Diet Assoc 102, 58-64.

32. Longbottom PJ, Wrieden WL \& Pine CM (2002) Is there a relationship between the food intakes of Scottish 
5(1/2)-8(1/2)-year-olds and those of their mothers? J Hum Nutr Diet 15, 271-279.

33. Oliveria SA, Ellison RC, Moore LL, et al. (1992) Parent-child relationships in nutrient intake: the Framingham Children's Study. Am J Clin Nutr 56, 593-598.

34. Vereecken CA, Keukelier E \& Maes L (2004) Influence of mother's educational level on food parenting practices and food habits of young children. Appetite 43, 93-103.

35. Bere E \& Klepp KI (2004) Correlates of fruit and vegetable intake among Norwegian schoolchildren: parental and selfreports. Public Health Nutr 7, 991-998.

36. Coon KA, Goldberg J, Rogers BL, et al. (2001) Relationships between use of television during meals and children's food consumption patterns. Pediatrics 107, E7.

37. Reilly JJ, Armstrong J, Dorosty AR, et al. (2005) Early life risk factors for obesity in childhood: cohort study. $B M J$ 330, 1357.
38. Bower JA \& Sandall L (2002) Children as consumerssnacking behaviour in primary school children. Int J Consum Stud 26, 15-26.

39. Serdula MK, Alexander MP, Scanlon KS, et al. (2001) What are preschool children eating? A review of dietary assessment. Annu Rev Nutr 21, 475-498.

40. Hu FB, Rimm E, Smith-Warner SA, et al. (1999) Reproducibility and validity of dietary patterns assessed with a foodfrequency questionnaire. Am J Clin Nutr 69, 243-249.

41. Ruston D, Hoare J, Henderson L, et al. (2004) The National Diet and Nutrition Survey: Adults Aged 19 to 64 Years. Vol. 4. Nutritional Status (Anthropometry and Blood Analytes), Blood Pressure and Physical Activity. London: The stationery Office.

42. Borland SE, Robinson SM, Crozier SR, et al. (2008) Stability of dietary patterns in young women over a 2-year period. Eur J Clin Nutr 62, 119-126. 